\title{
EDITORIAL
}

\section{Advancing the use of non-invasive brain stimulation through systematic data review}

\author{
Paul B. Fitzgerald (iD \\ Epworth Centre for Innovation in Mental Health, Epworth Healthcare and Monash University Department of Psychiatry, Camberwell, VIC, \\ Australia.
}

Despite decades of research attention, thousands of clinical trials and major investment by the pharmaceutical industry and independent research bodies, depression remains a major social health problem whose importance and impact have not diminished in recent years. The limitations of mainstream pharmaceutical and psychotherapeutic treatments are well documented, as are issues with medication-related side effects and overall acceptability for many patients. Thus, there continues to be a pressing need for new therapies, including increasing interest in the potential of various forms of non-invasive and invasive brain stimulation. Fortunately, during the last two decades a substantial body of research has led to a meaningful evidence base for several forms of brain stimulation, which is driving their use in clinical practice. However, this use remains patchy and there is resistance from clinicians and regulators in many parts of the world.

A clear illustration of this occurred in my own practice just a few weeks ago. I was talking to a patient, an intelligent and successful woman in her 50s who had been struggling with an episode of severe major depression for about four years. She was finally coming out of this episode after a course of high-frequency left-sided repetitive transcranial magnetic stimulation (rTMS). She was extremely pleased but also very angry. Her anger was directed at the prominent academic psychiatrist who, $2 \frac{1}{2}$ years earlier, told her that rTMS was a complete waste of time and, to use his words, "a sham." She felt she had wasted $2 \frac{1}{2}$ years on an endless cycle of unsuccessful antidepressants.

This is why a recent publication by Razza et al. ${ }^{1}$ is of importance. Clearly, many people in the psychiatric community have been unconvinced by the barrage of clinical trials, larger multisite trials, and numerous meta-analyses (e.g., Berlim et al. $^{2}$ and Mutz et al. ${ }^{3}$ ) and comparative analyses showing the relative efficacy of rTMS compared to other treatment options for depression. ${ }^{4}$ Clearly documenting the evidence for rTMS and other neurostimulation techniques is essential to determining their place in clinical practice. They should be included in clinical practice guidelines and influence the decisions of mental health care funding bodies. During the production of evidence-based guidelines, Razza et al. were motivated

Correspondence: Paul B. Fitzgerald, Epworth Healthcare, The Epworth Clinic, 888 Toorak Rd, Camberwell, VIC, Australia, 3004. E-mail: paul.fitzgerald@monash.edu

Submitted Jan 10 2021, accepted Jan 20 2021, Epub Feb 222021. by a desire to comprehensively understand the evidence for these techniques and chose to document evidence quality using the comprehensive and up-to-date GRADE framework, along with the AMSTAR-2 approach.

A number of important findings emerged from this analysis. The authors found substantial support for the most common form of rTMS therapy, i.e., high-frequency stimulation applied to the left dorsolateral prefrontal cortex, as well as for bilateral rTMS. The high quality of evidence for rTMS across response, remission and continuous outcome variables leaves no doubts about its efficacy.

Readers may be somewhat surprised by the strength of the evidence about transcranial direct current stimulation (tDCS). High-quality evidence has been found supporting the use of tDCS in depression across all three outcome areas (response, remission and continuous outcomes). However, it is important to point out that the analysis does not speak to the relative efficacy of tDCS vs. rTMS, since the vast majority of research on rTMS has been conducted in patients with treatment-resistant depression, which is not the case for tDCS. Nevertheless, there is substantial evidence that both therapeutic modalities are efficacious and are potentially applicable in two different phases of the illness cycle. tDCS may lend itself to homebased treatment early in the course of the illness, since these patients could lack the commitment necessary for daily rTMS treatment in a clinical environment that more treatment-resistant patients may have.

Regarding the role of electroconvulsive therapy, only limited conclusions could be drawn due to the small number of trials in a single meta-analysis on this treatment, which only reported continuous outcomes. In reality, this reflects the historical development of electroconvulsive therapy, which occurred before clinical trial methodology became essential for establishing new therapeutic modalities. This should not be seen as a critique of the effectiveness of electroconvulsive therapy. Clearly, different standards of evaluation should be applied to treatments developed prior to modern trial methods and for which there are many decades of meaningful historical use.

In summary, both the powerful pharmaceutical industry and the large and influential body of psychotherapists have contributed to the historical marginalization of therapeutic

How to cite this article: Fitzgerald PB. Advancing the use of noninvasive brain stimulation through systematic data review. Braz J Psychiatry. 2021;43:458-459. http://dx.doi.org/10.1590/1516-44462021-1742 
brain stimulation in mental health care. Systematic and rigorous data collection is essential for the advancement of this field and its potential impact on the lives of patients with challenging problems such as depression.

\section{Disclosure}

PBF has received research equipment from Medtronic, MagVenture A/S, and Brainsway Ltd.; and he is a founder of TMS Clinics Australia.

\section{References}

1 Razza LB, dos Santos LA, Borrione L, Bellini H, Branco LC, Cretaz E, et al. Appraising the effectiveness of electrical and magnetic brain stimulation techniques in acute major depressive episodes: an umbrella review of meta-analyses of randomized controlled trials. Braz J Psychiatry. 2021;43:514-24.

2 Berlim MT, Van den Eynde F, Daskalakis ZJ. A systematic review and meta-analysis on the efficacy and acceptability of bilateral repetitive transcranial magnetic stimulation (rTMS) for treating major depression. Psychol Med. 2013;43:2245-54.

3 Mutz J, Edgcumbe DR, Brunoni AR, Fu CH. Efficacy and acceptability of non-invasive brain stimulation for the treatment of adult unipolar and bipolar depression: a systematic review and meta-analysis of randomised sham-controlled trials. Neurosci Biobehav Rev. 2018;92:291-303.

4 Papadimitropoulou K, Vossen C, Karabis A, Donatti C, Kubitz N. Comparative efficacy and tolerability of pharmacological and somatic interventions in adult patients with treatment-resistant depression: a systematic review and network meta-analysis. Curr Med Res Opin. 2017;33:701-11. 